\title{
On the Complexity of Selecting Disjunctions in Integer Programming
}

\author{
Ashutosh Mahajan* $\quad$ Ted Ralphs ${ }^{\dagger}$
}

October 25, 2009

\begin{abstract}
The imposition of general disjunctions of the form " $\pi x \leq \pi_{0} \vee \pi x \geq \pi_{0}+1$ ", where $\pi, \pi_{0}$ are integer valued, is a fundamental operation in both the branch-and-bound and cuttingplane algorithms for solving mixed integer linear programs. Such disjunctions can be used for branching at each iteration of the branch-and-bound algorithm or to generate split inequalities for the cutting-plane algorithm. We first consider the problem of selecting a general disjunction and show that the problem of selecting an optimal such disjunction, according to specific criteria described herein, is $\mathcal{N} \mathcal{P}$-hard. We further show that the problem remains $\mathcal{N} \mathcal{P}$-hard even for binary programs or when considering certain restricted classes of disjunctions. We observe that the problem of deciding whether a given inequality is a split inequality can be reduced to one of the above problems, which leads to a proof that the problem is $\mathcal{N} \mathcal{P}$-complete.
\end{abstract}

\section{Introduction}

In this paper, we study the computational complexity of the problem of selecting "optimal" general disjunctions in the branch-and-bound and cutting-plane algorithms for solving mixed integer linear programs (MILPs). The motivation for studying the complexity of these problems is that the solution of such problems may be useful in selecting disjunctions for branching or for generating valid inequalities. For instance, these problems can, in principle, be solved at each node of the branchand-bound tree to select an "optimal" disjunction for partitioning the feasible region of that node. In an earlier paper [Mahajan and Ralphs, 2009], we showed by means of several experiments that the number of nodes in the branch-and-bound tree can be reduced significantly (by a factor of more than two for $75 \%$ of instances in the test) by employing such selection procedures. However, the time required to solve these problems, when formulated as straightforward optimization problems, using a generic solver, is prohibitively large. In this paper, we show that these problems in fact lie in the complexity class $\mathcal{N} \mathcal{P}$-hard, even for binary MILPs and even when certain restrictions are imposed on the structure of the disjunctions.

\footnotetext{
* Department of Industrial and Systems Engineering, Lehigh University, Bethlehem, PA 18015, asm4@lehigh.edu, http://coral.ie.lehigh.edu/ asm4

${ }^{\dagger}$ Department of Industrial and Systems Engineering, Lehigh University, Bethlehem, PA 18015, ted@lehigh.edu, http://coral.ie.lehigh.edu/ ${ }^{\text {ted }}$
} 
Before defining the problem of selecting an optimal disjunction, it is important to understand the role of disjunctions in the branch-and-bound and cutting-plane algorithms. Consider the mathematical program

$$
\begin{aligned}
\min c x & \\
\text { s.t. } A x & \geq b \\
x & \in \mathbb{Z}^{d} \times \mathbb{R}^{n-d},
\end{aligned}
$$

where $b \in \mathbb{Q}^{m}, c \in \mathbb{Q}^{n}, A \in \mathbb{Q}^{m \times n}$ are inputs and where the variables with indices $1,2, \ldots, d$ are constrained to be integer-valued. A particular case of the MILP (1) is when $n=d$, i.e., when all variables are constrained to be integers. Such an MILP is also known simply as an integer program (IP).

$$
\begin{aligned}
\min c x & \\
\text { s.t. } A x & \geq b \\
x & \in \mathbb{Z}^{n} .
\end{aligned}
$$

We will henceforth work with this particular case for notational convenience. The complexity results for the more general mixed integer case follow from our results. We now briefly describe the branch-and-bound and the cutting-plane algorithms, emphasizing the role played by disjunctions in both. Both these methods start by solving a linear programming (LP) relaxation of (IP):

$$
\min _{x \in \mathcal{P}} c x,
$$

where $\mathcal{P}=\left\{x \in \mathbb{R}^{n} \mid A x \geq b\right\}$. If the solution $x^{*} \in \mathcal{P}$ to the LP relaxation is a member of $\mathbb{Z}^{n}$, then $x^{*}$ is an optimal solution for (IP) and we are done. Otherwise, we need to refine our search by tightening the relaxation. How this search is undertaken in each algorithm is described next.

Branch-and-Bound Algorithms. In an LP-based branch-and-bound algorithm for solving (IP), if $x^{*} \notin \mathbb{Z}^{n}$, we determine a disjunction (usually binary) that is satisfied by all solutions to (IP) but not satisfied by $x^{*}$. Such a disjunction, referred to henceforth as a valid disjunction, divides the feasible region of (IP) into (usually disjoint) subsets. The algorithm can then be applied recursively until exhaustion to the subproblems associated with the subsets obtained after such a disjunction is imposed. For a more complete and formal description, see [Nemhauser and Wolsey, 1988]. Note that we use the term subproblem to refer to the restriction of the original instance (IP) resulting from the imposition of one or more branching disjunctions. These subproblems should not be confused with the associated problem of selecting a branching disjunction, which is formulated in the following sections.

A disjunction of the form " $x_{i} \leq k \vee x_{i} \geq k+1$ " for any $1 \leq i \leq n, k \in \mathbb{Z}$ is always valid for (IP). Such a disjunction will be called a variable disjunction. Most implementations of branch-and-bound use only variable disjunctions for branching. More generally, however, any $\pi \in \mathbb{Z}^{n}, \pi_{0} \in \mathbb{Z}$ yields a disjunction " $\pi x \leq \pi_{0} \vee \pi x \geq \pi_{0}+1$ ". Such a disjunction, referred to henceforth as a general disjunction and denoted by the ordered pair $\left(\pi, \pi_{0}\right)$, is also always valid. Since the set of general disjunctions includes all variable disjunctions, selecting a disjunction from this larger set should, in principle, be more effective. In this paper, we study the complexity of the problem of selecting an "optimal" general disjunction from this set. Since such general disjunctions can also be used to generate valid inequalities for cutting-plane algorithms, we describe these algorithms next. 
Cutting-Plane Algorithms. If the solution $x^{*} \in \mathcal{P}$ to the LP relaxation is not a member of $\mathbb{Z}^{n}$, then we can also determine a valid inequality, $(\alpha, \beta) \in \mathbb{R}^{n+1}$, such that $\alpha x^{*}<\beta$. An inequality $(\alpha, \beta) \in \mathbb{R}^{n+1}$ is said to be valid for problem (IP) if $\alpha x \geq \beta$ for any $x \in \mathcal{P} \cap \mathbb{Z}^{n}$. When such a valid inequality is violated by some solution to the LP relaxation, it is also known as a cutting plane. After such a cutting plane has been added to the LP relaxation of (IP), we can now solve the tighter relaxation again. When this process is applied iteratively, it is called a cutting-plane algorithm.

The first cutting-plane algorithm was proposed by Gomory [1958]. Since then, many different classes of valid inequalities have been discovered. The most general amongst them is the class of split inequalities which are inequalities obtained from the application of general disjunctions. Given a problem (IP) any inequality $(\alpha, \beta) \in \mathbb{R}^{n+1}$ is called an elementary split inequality [Cook et al., 1990] if, for some general disjunction $\left(\pi, \pi_{0}\right) \in \mathbb{Z}^{n+1},(\alpha, \beta)$ is valid for both sets: $\left\{x \in \mathcal{P} \mid \pi x \leq \pi_{0}\right\}$ and $\left\{x \in \mathcal{P} \mid \pi x \geq \pi_{0}+1\right\}$. In his survey, Cornuéjols [2008] points out that most classes of valid inequalities for solving general integer programs are particular cases of split inequalities.

Previous Work. In its simplest form, the efficiency of both the branch-and-bound and cuttingplane algorithms depends primarily on the number of iterations, i.e., the number of LP relaxations solved. The goal of selecting a disjunction can thus be viewed as that of minimizing the total number of iterations. Despite the close relationship between the branch-and-bound and cuttingplane algorithms, the question of which disjunctions to employ in each of the two algorithms has been studied largely in isolation. A disjunction for branching is usually evaluated by computing or estimating its effect on the objective function value of the LP relaxations of the subproblems. On the other hand, valid inequalities are usually selected on the basis of the violation of the current LP solution. Two notable exceptions are the computational studies of Karamanov and Cornuéjols [2007] and Cornuéjols et al. [2008] for selecting those disjunctions for branching that could be used for generating specific valid inequalities.

Liberatore [2000] showed in the context of Satisfiability Problems (SATs) that the problem of finding optimal variable disjunction (according to the criteria of minimizing the overall size of the search tree) for the branch-and-bound algorithm is $\mathcal{N} \mathcal{P}$-hard. Since SATs are reducible, in polynomial time, to IPs, a similar result may be expected for the case of IPs. In light of this observation, the problem of selecting an optimal general branching disjunction appears to be difficult. The approach taken by most solution procedures, and the one we shall take here, is then to evaluate candidate branching disjunctions by assessing their effect using more myopic criteria. Unfortunately, we show that the selection problems are $\mathcal{N} \mathcal{P}$-hard even when these criteria are used.

The primary criterion for selecting (for branch-and-bound) variable disjunctions studied in the literature so far is that of bound improvement. Both Linderoth and Savelsbergh [1999] and Achterberg et al. [2005] showed empirically that selecting a variable disjunction that leads to the maximum increase in the bound of the subproblems can reduce the number of subproblems required to solve the problem. Some other heuristic procedures to select variable disjunctions have been studied by, among others, Patel and Chinneck [2007] and Gilpin and Sandholm [2007].

On the other hand, the criteria for selecting general disjunctions for branching have primarily been limited to "width" of the feasible region associated with the LP relaxation of the current subproblem. In their survey, Aardal and Eisenbrand [2004] discussed the fact that when the dimension is fixed, polynomial time algorithms for solving integer programs can be obtained by branching on general disjunctions obtained by determining the so-called thin directions of the feasible region, 
i.e., disjunctions along which the width of the feasible region is small. These polynomial time algorithms are derived from the seminal work of Lenstra [H.W. Lenstra, 1983] and its extensions. It has also been shown, for instance by Krishnamoorthy and Pataki [2006], that certain specific problems can be solved "easily" if one branches on particular general disjunctions. Some heuristics that may enhance the computational efficiency of the branch-and-bound algorithm by branching on general disjunctions have also been proposed recently. Fischetti and Lodi [2003] proposed a primal heuristic in which the search space is constrained using a general disjunction. Owen and Mehrotra [2001] proposed a greedy heuristic to select a general disjunction for branching. Their criteria for selection is also the improvement in the associated bound.

Caprara and Letchford [2003] addressed the computational complexity of the problem of optimizing over the closure of elementary split inequalities and Balas and Saxena [2008] performed computational experiments to optimize over this closure. The elementary split closure of the problem (IP) is the feasible region obtained by adding all possible elementary split inequalities to the description of the LP relaxation. Cook et al. [1990] showed that this closure is polyhedral. Any inequality that is valid for this closure is said to have split-rank one. Therefore, it follows from the result of Caprara and Letchford [2003] that the problem of deciding if the split-rank of a given inequality is one is $\mathcal{N} \mathcal{P}$-complete. In this paper, we will also show that an inequality that has splitrank one may not be an elementary split inequality. We further show, that despite this difference, the problem of deciding if a given inequality is an elementary split inequality is also $\mathcal{N} \mathcal{P}$-complete.

The paper is structured as follows. In Section 2, we describe the criterion of maximizing bound improvement for selecting general disjunctions and also state our main result. We then discuss its relationship with the criterion of minimum width. The proofs of computational complexity of the problems are provided in Section 3. We then describe the implication of our results for two problems related to elementary split inequalities in Section 4. Finally, in Section 5, we present conclusions.

\section{Selecting General Disjunctions}

The complexity of the problem of finding a valid inequality that is most violated by a given vector has been studied previously for many classes of inequalities, including split inequalities. From the standpoint of formal complexity, the problem of selecting disjunctions for branching has received relatively less attention, yet there is a strong connection between the two problems in the case of split inequalities. In fact, as we discuss below, the problem of selecting a disjunction that maximizes the resulting bound improvement is equivalent to that of selecting an elementary split inequality by the same criterion. In what follows, we thus do not distinguish between the two cases, referring instead to the generic disjunction selection problem. We show how this problem relates to that of minimizing the width of a polytope and expand on the idea that the criterion of maximizing bound improvement leads to a unified view of the problem of selecting general disjunctions. Below, we formalize this problem and study its complexity in the next section.

\subsection{Maximizing Bound Improvement}

Consider the integer program (IP) and assume that the associated polyhedron $\mathcal{P}$ is nonempty. Let

$\left(\hat{\pi}, \hat{\pi}_{0}\right) \in \mathbb{Z}^{n+1}$ be a given general disjunction. Then the LPs associated with the subproblems 
created after branching are

$$
\begin{aligned}
& z_{L}^{*}=\min c x \quad z_{R}^{*}=\min c x \\
& \text { s.t. } A x \geq b \text { and s.t. } A x \geq b \\
& \hat{\pi} x \leq \hat{\pi}_{0} \quad \hat{\pi} x \geq \hat{\pi}_{0}+1 \text {. }
\end{aligned}
$$

The lower bound obtained for (IP) after solving these two LPs is $z_{l_{b}}=\min \left\{z_{L}^{*}, z_{R}^{*}\right\}$. We can also generate an elementary split inequality, say $(\hat{\alpha}, \hat{\beta}) \in \mathbb{R}^{n+1}$, using the same disjunction. The lower bound obtained after adding $(\hat{\alpha}, \hat{\beta})$ to the LP relaxation is

$$
\begin{array}{r}
z_{l_{c}}=\min c x \\
A x \geq b \\
\hat{\alpha} x \geq \hat{\beta} .
\end{array}
$$

The following proposition shows that the lower bound achieved by branching on a given disjunction is the same as the lower bound that may be achieved by generating elementary split inequalities from the same disjunction.

Proposition 2.1. Given (IP) and a valid disjunction $\left(\hat{\pi}, \hat{\pi}_{0}\right) \in \mathbb{Z}^{n+1}$. Let $z_{l_{b}}$ be the lower bound obtained after branching on $\left(\hat{\pi}, \hat{\pi}_{0}\right)$. Then

1. there exists an elementary split inequality that can be obtained from $\left(\hat{\pi}, \hat{\pi}_{0}\right)$ such that the lower bound obtained by adding it to the LP relaxation of $(I P)$ is $z_{l_{b}}$, and

2. the LP solution value after adding any elementary split inequality obtained from $\left(\hat{\pi}, \hat{\pi}_{0}\right)$ to the $L P$ relaxation of $(I P)$ is at most $z_{l_{b}}$.

Proof. Clearly, $c x \geq z_{l_{b}}$ is satisfied by all points of the sets $\left\{x \in \mathcal{P} \mid \hat{\pi} x \leq \hat{\pi}_{0}\right\}$ and $\{x \in \mathcal{P} \mid$ $\left.\hat{\pi} x \geq \hat{\pi}_{0}+1\right\}$. Therefore, $\left(c, z_{l_{b}}\right)$ is a valid elementary split inequality that when added to the LP relaxation makes the objective function value $z_{l_{b}}$.

Now consider any elementary split inequality, say $(\hat{\alpha}, \hat{\beta})$, derived from disjunction $\left(\hat{\pi}, \hat{\pi}_{0}\right)$, and let $z_{l_{c}}=\min \{c x \mid x \in \mathcal{P}, \hat{\alpha} x \geq \hat{\beta}\}$. From the definition of an elementary split inequality, $\min \left\{\hat{\alpha} x \mid x \in \mathcal{P}, \hat{\pi} x \leq \hat{\pi}_{0}\right\} \geq \hat{\beta}$ and thus the set $\left\{x \in \mathcal{P} \mid \hat{\pi} x \leq \hat{\pi}_{0}\right\} \subseteq\{x \in \mathcal{P} \mid \hat{\alpha} x \geq \hat{\beta}\}$. Hence, $\min \left\{c x \mid x \in \mathcal{P}, \hat{\pi} x \leq \hat{\pi}_{0}\right\} \geq z_{l_{c}}$. Similarly, $\min \left\{c x \mid x \in \mathcal{P}, \hat{\pi} x \geq \hat{\pi}_{0}+1\right\} \geq z_{l_{c}}$ and hence $z_{l_{b}} \geq z_{l_{c}}$.

Using the above mentioned equivalence we can now refer to the problems of maximizing the lower bound using either branching or an elementary split inequality together as a single problem of selecting general disjunctions.

Problem 1 (Greatest lower bound from a general disjunction). Given a mathematical program of the form (IP), find $\left(\hat{\pi}, \hat{\pi}_{0}\right) \in \mathbb{Z}^{n+1}$ such that $\min \left\{z_{L}^{*}, z_{R}^{*}\right\}$ is maximized, where $z_{L}^{*}, z_{R}^{*}$ are as defined in (3).

Problem 1 is an optimization problem and the associated decision problem is as follows.

Problem 2 (Lower bound from a general disjunction). Given a mathematical program of the form $(I P)$ and $z_{l} \in \mathbb{R}$, does there exist $\left(\hat{\pi}, \hat{\pi}_{0}\right) \in \mathbb{Z}^{n+1}$ such that the lower bound after imposing the disjunction is at least $z_{l}$, i.e., $\min \left\{z_{L}^{*}, z_{R}^{*}\right\} \geq z_{l}$ ? 
A special case of Problem 2 is one where the relaxation is required to become infeasible after the disjunction has been used. This means both problems in (3) become infeasible after a disjunction $\left(\hat{\pi}, \hat{\pi}_{0}\right)$ has been used for branching or an elementary split inequality $(\mathbf{0}, 1)$, where $\mathbf{0}$ is an $n$ dimensional vector of all zeros, can be derived imposing the disjunction.

Problem 3 (Disjunctive infeasibility). Given a mathematical program of the form (IP), does there exist $\left(\hat{\pi}, \hat{\pi}_{0}\right) \in \mathbb{Z}^{n+1}$ such that the feasible region of the LP relaxation of (IP) after imposing the disjunction is empty?

The solution to Problem 3 does not depend upon the cost vector $c$ because it is only desired to prove that the problem (IP) is infeasible. The problem of finding a desired disjunction $\left(\hat{\pi}, \hat{\pi}_{0}\right)$ can be formulated as follows. Assume again that $\mathcal{P}$ is nonempty. Suppose $\left(\hat{\pi}, \hat{\pi}_{0}\right)$ is chosen such that both LPs (3) become infeasible. Then consider the following problems:

$$
\begin{aligned}
& \zeta_{L}^{*}=\min \hat{\pi} x \quad \text { and } \quad \zeta_{R}^{*}=\min -\hat{\pi} x \\
& \text { s.t. } A x \geq b \text { and s.t. } A x \geq b \text {. }
\end{aligned}
$$

The dual of each of the above two programs can be written, respectively, as:

$$
\begin{aligned}
& \zeta_{L}^{*}=\max p b \quad \zeta_{R}^{*}=\max q b \\
& \text { s.t. } p A=\hat{\pi} \text { and s.t. } q A=-\hat{\pi} \\
& p \geq 0 \quad q \geq 0 .
\end{aligned}
$$

The programs (3) are infeasible if and only if $\zeta_{L}^{*}>\hat{\pi}_{0}$ and $\zeta_{R}^{*}>-\left(\hat{\pi}_{0}+1\right)$. By using this condition and combining the above two dual formulations, one can get the desired formulation for giving an answer to Problem 3. More precisely, the LPs (3) are infeasible if and only if the system

$$
\begin{aligned}
p A-\pi & =0 \\
q A+\pi & =0 \\
p b-\pi_{0} & >0 \\
q b+\pi_{0} & >-1 \\
p & \geq 0 \\
q & \geq 0 \\
\left(\pi, \pi_{0}\right) & \in \mathbb{Z}^{n+1},
\end{aligned}
$$

has a feasible solution with $\pi=\hat{\pi}, \pi_{0}=\hat{\pi}_{0}$. Hence, there is a disjunction that proves infeasibility if and only if there is a solution to (7).

Once we have a formulation that may be solved in order to answer Problem 3, we can extend it (by adding the constraint $c x \leq z_{l}$ to the original set of constraints $A x \geq b$ and following the above steps) to address Problem 2 as well. One can also answer Problem 1 by iterating this process over different values of $z_{l}$. More details about this procedure are described in Mahajan and Ralphs [2009].

The main result of this paper is that Problem 3 is $\mathcal{N} \mathcal{P}$-complete. We defer the proof until Section 3, where we also present proofs for the complexity of other restrictions of the problem. Problem 3 is equivalent to that of finding $\left(\hat{\pi}, \hat{\pi}_{0}\right) \in \mathbb{Z}^{n+1}$ such that $\mathcal{P} \subseteq\left\{x \in \mathbb{R}^{n} \mid \hat{\pi}_{0}<\hat{\pi} x<\hat{\pi}_{0}+1\right\}$. If such a $\left(\hat{\pi}, \hat{\pi}_{0}\right)$ exists, then the "width" $\mathcal{P}$ is less than one. In the next section, we study the problem of minimizing the width of $\mathcal{P}$ and show that the general framework described above can be extended to that problem as well. 


\section{$2.2 \quad$ Minimizing Width}

Assuming that $\mathcal{P}$ is full dimensional, the width of $\mathcal{P}$ in direction $\pi \in \mathbb{Z}^{n}$ is $\max _{x, y \in \mathcal{P}}(\pi x-\pi y)$, while the width of $\mathcal{P}$ is

$$
w(\mathcal{P})=\min _{\pi} \max _{x, y \in \mathcal{P}}(\pi x-\pi y), \pi \in \mathbb{Z}^{n}, \pi \neq 0 .
$$

A vector $\pi$ that is obtained from the above optimization problem, along with a scalar $\pi_{0}=\left\lfloor\pi x^{*}\right\rfloor$, where $x^{*}$ is the optimal solution of the LP relaxation (2), can then be used to determine a disjunction for branching. Sebö [1999] showed that the problem of determining whether $w(\mathcal{P}) \leq 1$ is $\mathcal{N} \mathcal{P}$ complete, even when $\mathcal{P}$ is a simplex. It is also known, from a result of Banaszczyk et al. [1999], that if $\mathcal{P} \cap \mathbb{Z}^{n}$ is empty, then $w(\mathcal{P}) \leq C n^{\frac{3}{2}}$, where $C$ is a constant. Derpich and Vera [2006] approximate the direction of the minimum width in order to assign priorities for branching on variables and use this to select variable disjunctions.

The width of $\mathcal{P}$ in a given direction $\hat{\pi}$ can be obtained by solving the LP

$$
\begin{aligned}
\max & \hat{\pi} x-\hat{\pi} y \\
\text { s.t. } & A x \geq b \\
& A y \geq b .
\end{aligned}
$$

The dual associated with the LP (8) is

$$
\begin{array}{cl}
\min & -q b-p b \\
\text { s.t. } & p A=\hat{\pi} \\
& q A=-\hat{\pi} \\
& p, q \geq 0 .
\end{array}
$$

Therefore, the problem of finding $w(\mathcal{P})$ can be written as

$$
\begin{array}{ll}
\min & -q b-p b \\
\text { s.t. } & p A-\pi=0 \\
& q A+\pi=0 \\
& \pi \neq 0 \\
& \pi \in \mathbb{Z}^{n} \\
& p, q \geq 0 .
\end{array}
$$

Since the disjunction $\left(\pi, \pi_{0}\right)$ is the same as the disjunction $\left(-\pi,-\pi_{0}-1\right)$, the condition $\pi \neq 0$ can be replaced by the inequality $\sum_{i=1}^{n} \pi_{i} \geq 1$. Problem (10) can now be solved as an IP.

Note that if there exists a $\left(\hat{\pi}, \hat{\pi}_{0}\right) \in \mathbb{Z}^{n+1}$ that satisfies the formulation $(7)$, then $w(\mathcal{P})<1$. However, the converse is not true. To see this, consider as an example $\mathcal{P}=\left\{x \in \mathbb{R}_{+}^{2} \mid 3 \leq\right.$ $\left.4 x_{1}+4 x_{2} \leq 5\right\}$ and $n=2$. Then, even though $w(\mathcal{P}) \leq \frac{1}{2}<1$, (IP) is still feasible. Comparing formulations (7) and (10), one can see that (7) is more constrained than (10). As a result, there may be some benefit to using solutions to the formulation (7) to generate branching disjunctions over those of (10). A feasible solution to formulation (7) guarantees that the LP relaxations associated with both subproblems created after branching are infeasible and therefore gives a short proof of infeasibility, provided that such a short proof exists. Branching along a direction of minimum width does not guarantee this. As an example, consider an IP with feasible region 
$\left\{x \in \mathbb{Z}_{+}^{2} \mid 7 \leq 8 x_{1}+8 x_{2} \leq 9,-3 \leq 4 x_{1}-4 x_{2} \leq 3\right\}$. Branching on the disjunction $x_{1} \leq 0 \vee x_{1} \geq 1$ immediately makes LP relaxation of each subproblem infeasible while branching along a direction of minimum width $(w(\mathcal{P})=0.25), x_{1}+x_{2} \geq 2 \vee x_{1}+x_{2} \leq 1$ results in two subproblems out of which one still has a feasible LP relaxation and needs further processing. Krishnamoorthy [2008] showed that, in general, branching along a direction of minimum width need not result in a small branch-and-bound tree, even in higher dimensions.

Even though there are some similarities in the formulations (7) and (10), it is not easy to reduce the problem of finding $w(\mathcal{P})$ to Problem 3 . Therefore, we use a different approach to address the complexity of the latter.

\section{Complexity of Selecting General Disjunctions}

In this section, we begin by showing that Problem 3 lies in the complexity class $\mathcal{N} \mathcal{P}$. For the case when $\left(\pi, \pi_{0}\right)$ is restricted to variable disjunctions only, Problem 3 can be solved in time polynomial in the size of the input by solving the two LPs associated with each of the $n$ possible variable disjunctions. The following proofs show that the problem becomes difficult in the case of general disjunctions. We show that Problem 3 is $\mathcal{N} \mathcal{P}$-complete by reducing the well known number-partitioning problem to Problem 3 . We then show that the problem remains $\mathcal{N} \mathcal{P}$-complete even when several common restrictions are introduced. We start with the following lemma.

Lemma 3.1. If $\left(\hat{\pi}, \hat{\pi}_{0}, \hat{p}, \hat{q}\right)$ is a feasible solution to (7), then $\hat{\pi}_{0}<\hat{p} b \leq-\hat{q} b<\hat{\pi}_{0}+1$.

Proof. The first and last inequalities come directly from the formulation (7). Let $\zeta_{L}^{*}=\min _{x}\{\hat{\pi} x \mid$ $A x \geq b\}, \zeta_{R}^{*}=\max _{x}\{\hat{\pi} x \mid A x \geq b\}$. Then $\zeta_{L}^{*} \leq \zeta_{R}^{*}$. Also, $p=\hat{p}$ and $q=\hat{q}$ are feasible solutions to the dual programs (6). By using weak duality on the associated LPs (5), we get that $\zeta_{L}^{*} \geq \hat{p} b$ and $\zeta_{R}^{*} \leq-\hat{q} b$. Thus, $\hat{\pi}_{0}<\hat{p} b \leq \zeta_{L}^{*} \leq \zeta_{R}^{*} \leq-\hat{q} b<\hat{\pi}_{0}+1$.

We now show that Problem 3 is in the complexity class $\mathcal{N} \mathcal{P}$. If the matrices $A, b$ have integer entries only, then we claim that constraints $p<\mathbf{1}$, where $\mathbf{1}$ is the vector of all ones, may be added to (7) without any loss of generality. In order to see this, suppose the formulation (7) has a feasible solution with $p=\hat{p}, q=\hat{q}, \pi=\hat{\pi}, \pi_{0}=\hat{\pi}_{0}$. Further suppose that $\hat{p}_{i} \geq 1$ for some $i, 1 \leq i \leq m$. Then $p=\hat{p}-e_{i}, q=\hat{q}+e_{i}, \pi=\hat{\pi}-a_{i}, \pi_{0}=\hat{\pi}_{0}-b_{i}$ is also a feasible solution. Here, $e_{i}$ is the $i^{t h}$ unit vector and $a_{i}$ the $i^{\text {th }}$ row of the matrix $A$. This process can be applied repeatedly until $p$ is component-wise less than 1. If we assume that $p<\mathbf{1}$, then $|p b| \in\left[0, \sum_{i=1}^{m}\left|b_{i}\right|\right)$. Also, using (7), $\left|\pi_{j}\right| \in\left[0, \sum_{i=1}^{m}\left|a_{i j}\right|\right), j=1, \ldots, n$. Using Lemma 3.1, this implies $\left|\pi_{0}\right| \leq|p b| \leq \sum_{i=1}^{m}\left|b_{i}\right|$. So, if the system (7) is feasible, then a feasible solution may be expressed in size that is polynomial in the size of the input. Also, given a $\left(\hat{\pi}, \hat{\pi}_{0}\right)$, one can determine whether a disjunction on $\left(\hat{\pi}, \hat{\pi}_{0}\right)$ will make the LPs (3) infeasible in time that is polynomial in the size of the input by solving the two linear programs. This shows that Problem 3 lies in the complexity class $\mathcal{N} \mathcal{P}$.

Before further addressing the complexity of Problem 3, we consider the same problem applied to a system of linear Diophantine equations in place of the system of form (IP). Suppose we are given a system of linear Diophantine equations of the form,

$$
\begin{aligned}
& A x=b \\
& x \in \mathbb{Z}^{n} .
\end{aligned}
$$


Such equations can be solved in time polynomial in the size of the input [Nemhauser and Wolsey, 1988, pg. 191]. A branching disjunction $\left(\hat{\pi}, \hat{\pi}_{0}\right)$ that can make the associated LP relaxations of (11) infeasible can be shown, by using the approach above, to satisfy (along with a suitable $\hat{p}, \hat{q}$ ) the system

$$
\begin{aligned}
p A & =\pi, \\
-q A & =\pi, \\
p b & >\pi_{0}, \\
-q b & <1+\pi_{0}, \text { and } \\
\left(\pi, \pi_{0}\right) & \in \mathbb{Z}^{n+1} .
\end{aligned}
$$

We claim that the system (12) can be solved in time polynomial in the size of the input. The system of Diophantine equations (11) is infeasible if and only if there exists a $\lambda$ such that $\lambda A \in \mathbb{Z}^{m}$ and $\lambda b \notin \mathbb{Z}$ [Nemhauser and Wolsey, 1988, pg. 191]. Further, if (11) is infeasible, then such a $\lambda$ can be found in polynomial time. In such a case, $p=-q=\lambda, \pi=\lambda A, \pi_{0}=\lfloor\lambda b\rfloor$ is a feasible solution to (12). Conversely, suppose that (11) has a feasible solution $x^{0}$. Such a feasible solution can be found in polynomial time. Then for any $\left(\pi, \pi_{0}\right) \in \mathbb{Z}^{n+1}, \pi x^{0}=p A x^{0}=p b$ and $\pi x^{0}=-q A x^{0}=-q b$. Since $\pi x^{0} \in \mathbb{Z}$, there is no $\pi_{0} \in \mathbb{Z}$ such that $\pi_{0}>\pi x^{0}$ and $\pi_{0}<\pi x^{0}+1$. Thus, in this case, the existence of the solution $x^{0}$ is sufficient to show that (12) is infeasible. So a feasible solution for the system (12) can be found or it can be shown that no such solution exists in time polynomial in the size of the input.

Now consider the problem (IP) again. We just have shown that the problem of finding $\lambda \in \mathbb{R}^{m}$ such that $\lambda A \in \mathbb{Z}^{n}, \lambda b \notin \mathbb{Z}$ is polynomially solvable. Existence of such a $\lambda$ is a necessary condition for the feasibility of a given program of the form (7). To see this, suppose $p=\hat{p}, \pi=\hat{\pi}, \pi_{0}=\hat{\pi}_{0}$ are feasible for (7) and substitute $\lambda=\hat{p}$. Then $\lambda A=\hat{\pi} \in \mathbb{Z}^{n}$, but $\hat{\pi}_{0}<\lambda b<\hat{\pi}_{0}+1$. Existence of such a $\lambda$ is not, however, sufficient for feasibility of (7). For instance, consider the set: $\mathcal{P} \cap \mathbb{Z}^{2}=$ $\left\{x \in \mathbb{Z}^{2} \mid 3 x_{1}+6 x_{2} \geq 2\right\}$ and $\lambda=\frac{1}{3}$. Clearly $\lambda A \in \mathbb{Z}^{2}, \lambda b \notin \mathbb{Z}$. Still, $\mathcal{P} \cap \mathbb{Z}^{n}$ has at least one feasible point $(1,0)$. This provides a hint that Problem 3 may not be solvable in polynomial time.

We now show that Problem 3 is $\mathcal{N} \mathcal{P}$-complete by reducing the well-known number partitioning problem to Problem 3. The Number Partitioning Problem PARTITION is defined as follows

Problem 4 (PARTITION, [Garey and Johnson, 1979]). Given a finite set $S$ and a size $a^{i} \in \mathbb{Z}_{+}$ for each $i \in S$. Is there a subset $K \subseteq S$ such that $\sum_{i \in K} a^{i}=\sum_{i \in S \backslash K} a^{i}$ ?

Proposition 3.2. Problem 3 is $\mathcal{N} \mathcal{P}$-complete.

Proof. The proof is a modification of the approach used by Sebő [1999] for the problem of finding width of the LP relaxation. Consider Problem 4 defined above, which is known to be $\mathcal{N} \mathcal{P}$-complete. Let $n \in \mathbb{N}, S=\{1,2, \ldots,(n-1)\}, a^{i} \in \mathbb{Z}_{+}, i \in S$ be inputs for Problem 4. Let $s=\frac{1}{2} \sum_{i \in S} a^{i}$. An instance of Problem 4 can be answered "yes" if and only if there exists a set $K \subseteq\{1,2, \ldots, n-1\}$ such that $\sum_{i \in K} a^{i}=s$. Since multiplying each $a^{i}$ by 4 results in a problem equivalent to Problem 4 , it is assumed, without loss of generality, that $s \in \mathbb{Z}_{+}, s \geq 2$. Problem 4 can be reduced to Problem 3 as follows. Consider the simplex $\mathcal{P}_{s}$ of points $v^{i}, i=1 \ldots n+1$ in $n$ dimensions, with the coordinates 
of $v^{i}$ defined as

$$
v_{j}^{i}= \begin{cases}\frac{1}{2 n} & \text { if } j \neq i, i=1,2, \ldots, n, \\ \frac{1}{2 n}+\frac{1}{2} & \text { if } j=i, i=1,2, \ldots, n, \\ a^{j} & \text { if } i=n+1, j=1,2, \ldots, n-1, \\ -\frac{1}{2} \sum_{k=1}^{n-1} a^{k}+\frac{1}{2} & \text { if } i=n+1, j=n .\end{cases}
$$

So, $v^{1}=\left(\frac{1}{2 n}+\frac{1}{2}, \frac{1}{2 n}, \frac{1}{2 n}, \ldots, \frac{1}{2 n}\right), v^{2}=\left(\frac{1}{2}, \frac{1}{2}+\frac{1}{2 n}, \frac{1}{2 n}, \ldots, \frac{1}{2 n}\right), \ldots, v^{n}=\left(\frac{1}{2}, \frac{1}{2}, \ldots, \frac{1}{2 n}+\frac{1}{2}\right), v^{n+1}=$ $\left(a^{1}, a^{2}, \ldots, a^{n-1},-s+\frac{1}{2}\right)$. We will show that the desired subset $K$ exists if and only if there exists $\left(\hat{\pi}, \hat{\pi}_{0}\right) \in \mathbb{Z}^{n+1}$, such that $\mathcal{P}_{s} \subseteq\left\{x \mid \hat{\pi}_{0}<\hat{\pi} x<\hat{\pi}_{0}+1\right\}$.

Suppose the desired subset $K$ exists, i.e., $K$ is a set such that $\sum_{i \in K} a^{i}=s$. Let $\hat{\pi}_{i}=1, i \in K$, $\hat{\pi}_{n}=1, \hat{\pi}_{i}=0, i \notin K \cup\{n\}, \hat{\pi}_{0}=0$. Then, $0<\hat{\pi} v^{i}<1, i=1,2, \ldots, n$. Also, $v^{n+1} \hat{\pi}=\frac{1}{2}$. Since all vertices of $\mathcal{P}_{s}$ satisfy the condition $\hat{\pi}_{0}<\hat{\pi} x<\hat{\pi}_{0}+1, \mathcal{P}_{s} \subseteq\left\{x \mid \hat{\pi}_{0}<\hat{\pi} x<\hat{\pi}_{0}+1\right\}$. $\left(\hat{\pi}, \hat{\pi}_{0}\right)$ is then the required disjunction.

Conversely, suppose there exists some $\left(\hat{\pi}, \hat{\pi}_{0}\right) \in \mathbb{Z}^{n+1}$ such that $\mathcal{P}_{s} \subseteq\left\{x \mid \hat{\pi}_{0}<\hat{\pi} x<\hat{\pi}_{0}+1\right\}$. Then, $\hat{\pi}_{0}<\hat{\pi} v^{i}<\hat{\pi}_{0}+1, i=1,2, \ldots,(n+1)$ and $\left|\hat{\pi}\left(v^{i}-v^{k}\right)\right|<1, i=1,2, \ldots, n, k=1,2, \ldots, n$. Substituting the coordinates of $v^{i}$ and $v^{k}$, one gets that $\left|\frac{\hat{\pi}_{i}-\hat{\pi}_{k}}{2}\right|<1$. Since $\hat{\pi}_{i}, \hat{\pi}_{k} \in \mathbb{Z}$, this means that $\left|\hat{\pi}_{i}-\hat{\pi}_{k}\right| \leq 1$ for each pair $(i, k) \in\{1,2, \ldots, n\}^{2}$. So, $\hat{\pi}_{i} \in\{t, t+1\}, i=1,2, \ldots, n$ for some $t \in \mathbb{Z}$. Since disjunction $\left(\hat{\pi}, \hat{\pi}_{0}\right)$ is equivalent to disjunction $\left(-\hat{\pi},-\hat{\pi}_{0}-1\right)$, it can be assumed without loss of generality that $t \geq 0$. Let $K=\left\{i \mid \hat{\pi}_{i}=t+1\right\}$. Substituting the coordinates of $v^{1}$ and $\hat{\pi}$ into the inequalities $\hat{\pi}_{0}<v^{1} \hat{\pi}<\hat{\pi}_{0}+1$, one gets

$$
\begin{aligned}
& \hat{\pi}_{0}<\quad \quad \sum_{i=1}^{n} v_{i}^{1} \hat{\pi}_{i}<\hat{\pi}_{0}+1 \\
& \Rightarrow \hat{\pi}_{0}<\quad \sum_{i=1}^{n} \frac{\hat{\pi}_{i}}{2 n}+\frac{\hat{\pi}_{1}}{2}<\hat{\pi}_{0}+1 \\
& \Rightarrow \hat{\pi}_{0}<\frac{t}{2}+\sum_{i \in K} \frac{1}{2 n}+\frac{\hat{\pi}_{1}}{2}<\hat{\pi}_{0}+1 .
\end{aligned}
$$

Since $\frac{\hat{\pi}_{1}}{2} \in\left\{\frac{t}{2}, \frac{t+1}{2}\right\}$, the only integer value of $\hat{\pi}_{0}$ that satisfies the above condition is $\hat{\pi}_{0}=t$. Thus $\hat{\pi} \in\{t, t+1\}^{n}, \hat{\pi}_{0}=t$. Also, $K=\phi$ would mean that $\hat{\pi}_{0}<t<\hat{\pi}_{0}+1$. This is not possible for any integers $t, \hat{\pi}_{0}$. Hence $K$ is not empty. The condition $\hat{\pi}_{0}<\hat{\pi} v^{n+1}<\hat{\pi}_{0}$ implies:

$$
\begin{array}{rr} 
& \sum_{i=1}^{n} v_{i}^{n+1} \hat{\pi}_{i}<\hat{\pi}_{0}+1 \\
\Rightarrow \hat{\pi}_{0}<\quad & \sum_{i=1}^{n-1} \hat{\pi}_{i} a^{i}-\hat{\pi}_{n} s+\frac{\hat{\pi}_{n}}{2}<\hat{\pi}_{0}+1 \\
\Rightarrow t<t \sum_{i=1}^{n-1} a^{i}+\sum_{i \in K} a^{i}-\hat{\pi}_{n} s+\frac{\hat{\pi}_{n}}{2}<t+1 & 2 t s+\sum_{i \in K} a^{i}-\hat{\pi}_{n} s+\frac{\hat{\pi}_{n}}{2}<t+1 .
\end{array}
$$


Now there are two cases. Suppose $\hat{\pi}_{n}=t$. Then the above condition implies that $t<t s+\sum_{i \in K} a^{i}+$ $\frac{t}{2}<t+1$. This is not possible because $s \geq 2$ and $K \neq \phi$. Thus, $\hat{\pi}_{n}$ must equal $t+1$. In this case, the above condition becomes:

$$
\begin{aligned}
& t<2 t s+\sum_{i \in K} a^{i}-t s-s+\frac{t+1}{2}<t+1 \\
& \Rightarrow t<\quad(t-1) s+\sum_{i \in K} a^{i}+\frac{t+1}{2}<t+1 .
\end{aligned}
$$

Since $s \geq 2$ and $K \neq \phi$, the only value that $t$ may assume is $t=0$. That means $0<\sum_{i \in K} a^{i}-s+\frac{1}{2}<$ 1. Thus $\sum_{i \in K} a^{i}=s$ and $K$ is the required subset for Problem 4.

Thus, given a simplex $\mathcal{P}_{s}$, the problem of finding $\left(\hat{\pi}, \hat{\pi}_{0}\right) \in \mathbb{Z}^{n+1}$ is $\mathcal{N} \mathcal{P}$-complete. Since $\mathcal{P}_{s}$ is a simplex, its description can be transformed into form (IP) in time polynomial in the size of the description of $\mathcal{P}_{s}$. This completes the required proof.

Even though the above proof did not assume any restrictions on values of $\left(\hat{\pi}, \hat{\pi}_{0}\right)$, the reduction from Problem 4 imposed the conditions $\hat{\pi} \in\{0,1\}^{n}$. This shows that several restrictions of Problem 3 are also $\mathcal{N} \mathcal{P}$-complete. Some of these are listed below

Proposition 3.3. The following restrictions of Problem 3 are $\mathcal{N} \mathcal{P}$-complete.

1. Given a mathematical program of the form $(I P)$, does there exist $\left(\hat{\pi}, \hat{\pi}_{0}\right) \in\{0,1\}^{n+1}$ such that both the subproblems (3) created after branching on $\left(\hat{\pi}, \hat{\pi}_{0}\right)$ are infeasible.

2. Given a mathematical program of the form (IP), does there exist $\hat{\pi} \in\{0,1\}^{n}$ such that both the subproblems (3) created after branching on $(\hat{\pi}, 0)$ are infeasible.

3. Given a mathematical program of the form (IP), does there exist $\hat{\pi} \in\{0,1\}^{n}, \hat{\pi}_{0} \in \mathbb{Z}$ such that both the subproblems (3) created after branching on $\left(\hat{\pi}, \hat{\pi}_{0}\right)$ are infeasible.

4. Given a mathematical program of the form (IP), does there exist $\hat{\pi} \in \mathbb{Z}_{+}^{n}$ such that both the subproblems (3) created after branching on $(\hat{\pi}, 0)$ are infeasible.

5. Given a mathematical program of the form (IP), does there exist $\hat{\pi} \in \mathbb{Z}^{n}$ such that both the subproblems (3) created after branching on $(\hat{\pi}, 0)$ are infeasible.

6. Given a mathematical program of the form (IP), does there exist $\hat{\pi} \in\{0,1,-1\}^{n}, \hat{\pi}_{0} \in \mathbb{Z}$ such that both the subproblems (3) created after branching on $\left(\hat{\pi}, \hat{\pi}_{0}\right)$ are infeasible. (This problem is mentioned because Owen and Mehrotra [2001] developed a greedy heuristic for the optimization version of this problem, without addressing the complexity of the problem).

Proof. The proof of each of the above propositions follows directly from the proof of Proposition 3.2 above.

If $Q$ is a polytope, then the fact that $Q \subseteq\left\{x \mid \hat{\pi}_{0}<\hat{\pi} x<\hat{\pi}_{0}+1\right\}$, for some $\left(\hat{\pi}, \hat{\pi}_{0}\right) \in \mathbb{Z}^{n+1}$, is sufficient to show that $w(Q)<1$. The proof provided above settles the question of complexity of 
finding such a sufficient condition. If a program of form (IP) has only binary variables, i.e., it is of the form:

$$
\begin{gathered}
\min c x \\
\text { s.t. } A x \geq b \\
x \in\{0,1\}^{n},
\end{gathered}
$$

then the width of the associated polyhedron $\mathcal{P}_{b}$ is trivially at most one. The following proposition shows that the problem of deciding whether there exists a disjunction $\left(\hat{\pi}, \hat{\pi}_{0}\right)$ that will prove the infeasibility of a binary program is also $\mathcal{N} \mathcal{P}$-complete.

Problem 5 (Disjunctive infeasibility for binary programs). Given a mathematical program of the form $\left(P_{b}\right)$, does there exist a disjunction $\left(\hat{\pi}, \hat{\pi}_{0}\right) \in \mathbb{Z}^{n+1}$, that proves infeasibility?

Problem 5 is a special case of Problem 3 and hence the proof of $\mathcal{N} \mathcal{P}$-completeness of the latter follows from that of the former. However, we address the complexity of Problem 5 separately because the proof is easier to understand having seen that of Problem 3.

Proposition 3.4. Problem 5 is $\mathcal{N} \mathcal{P}$-complete.

Proof. The proof is similar to that of Proposition 3.2. Let $n \in \mathbb{Z}_{+}, S=\{1,2, \ldots,(n-1)\}, a^{i} \in$ $\mathbb{Z}_{+}, i \in S$ be inputs for an instance of Problem 4 . We need to modify our previous transformation because coordinates of the feasible region of $\mathcal{P}$ can only lie in $[0,1]$, while $a^{i} \in \mathbb{Z}_{+}, i \in S$. Let $M=\sum_{i \in S} a^{i}$ and $m=\frac{1}{M}$. If each $a^{i}, i \in S$ is divided by $M$, then the problem 4 can be transformed into an equivalent problem as follows. Let $\tilde{a}^{i}\left(=\frac{a^{i}}{M}\right) \in \mathbb{Q}_{+}, i \in S$ so that $\sum_{i \in S} \tilde{a}^{i}=1$. The answer to an instance of Problem 4 is "yes" if and only if there exists a set $K \subseteq\{1,2, \ldots, n-1\}$ such that $\sum_{i \in K} \tilde{a}^{i}=\frac{1}{2}$. Since each $\tilde{a}^{i}$ is an integer multiple of $\frac{1}{M}$, there is no $K \subseteq S$ such that $\sum_{i \in K} \tilde{a}^{i} \in\left[\frac{1}{2}-\frac{1}{2 M}, \frac{1}{2}\right)$ or $\sum_{i \in K} a^{i} \in\left(\frac{1}{2}, \frac{1}{2}+\frac{1}{2 M}\right]$. This observation will be useful later.

Problem 4 can now be reduced to Problem 5 as follows. Let $\epsilon=\frac{1}{2 M}=\frac{m}{2}$. Consider the convex hull, $\mathcal{P}_{s}$, of points $v^{i}, i=1 \ldots n+3$ in $n+1$ dimensions, where the coordinates of $v^{i}$ are defined as

$$
v_{j}^{i}= \begin{cases}\frac{1}{2 n} & \text { if } j \neq i, j \neq n, j \neq n+1, i=1,2, \ldots, n-1 \\ \frac{1}{2 n}+\frac{1}{2} & \text { if } j=i, i=1,2, \ldots, n-1 \\ 0 & \text { if } j=n, n+1, i=1,2, \ldots, n-1 \\ \tilde{a}^{j} & \text { if } j=1,2, \ldots, n-1, i=n \\ 1 & \text { if } j=n, n+1, i=n \\ \tilde{a}^{j} & \text { if } j=1,2, \ldots, n-1, i=n+1 \\ \frac{1}{2}-\epsilon & \text { if } j=n, i=n+1 \\ 0 & \text { if } j=n+1, i=n+1 \\ \tilde{a}^{j} & \text { if } j=1,2, \ldots, n-1, i=n+2 \\ 0 & \text { if } j=n, i=n+2 \\ \frac{1}{2}-\epsilon & \text { if } j=n, i=n+2 \\ \frac{1}{2} & \text { if } j=n, i=n+3 \\ 0 & \text { if } j \neq n, i=n+3\end{cases}
$$


This means $v^{1}=\left(\frac{1}{2 n}+\frac{1}{2}, \frac{1}{2 n}, \frac{1}{2 n}, \ldots, 0,0\right), v^{2}=\left(\frac{1}{2 n}, \frac{1}{2 n}+\frac{1}{2}, \frac{1}{2 n}, \frac{1}{2 n}, \ldots, 0,0\right)$ etc. $v^{n-1}=\left(\frac{1}{2 n}, \ldots, \frac{1}{2 n}+\right.$ $\left.\frac{1}{2}, 0,0\right), v^{n}=\left(\tilde{a}_{1}, \tilde{a}_{2}, \ldots, \tilde{a}_{n-1}, 1,1\right), v^{n+1}=\left(\tilde{a}_{1}, \ldots, \tilde{a}_{n-1}, \frac{1}{2}-\epsilon, 0\right), v^{n+2}=\left(\tilde{a}_{1}, \ldots, \tilde{a}_{n-1}, 0, \frac{1}{2}-\epsilon\right)$, $v^{n+3}=\left(0,0, \ldots, 0, \frac{1}{2}, 0\right)$. Clearly, $\mathcal{P}_{s} \subseteq\left\{x \in \mathbb{R}^{n+1} \mid 0 \leq x_{i} \leq 1, i=1,2, \ldots, n\right\}$. It will now be shown that a $K \subseteq S$ such that $\sum_{i \in K} \tilde{a}^{i}=\frac{1}{2}$ exists if and only if there exists $\left(\hat{\pi}, \hat{\pi}_{0}\right) \in \mathbb{Z}^{n+1}$, such that $\mathcal{P}_{s} \subseteq\left\{x \mid \hat{\pi}_{0}<\hat{\pi} x<\hat{\pi}_{0}+1\right\}$. Suppose $K \subseteq S$ such that $\sum_{i \in K} \tilde{a}^{i}=\frac{1}{2}$. Let $\hat{\pi}_{i}=1, i \in K$, $\hat{\pi}_{n}=1, \hat{\pi}_{n+1}=-1, \hat{\pi}_{i}=0, i \notin K \cup\{n, n+1\}, \hat{\pi}_{0}=0$. Then, $0<\hat{\pi} v^{i}<1, i=1,2, \ldots, n+3$. Since all vertices of $\mathcal{P}_{s}$ satisfy the condition $\hat{\pi}_{0}<\hat{\pi} x<\hat{\pi}_{0}+1, \mathcal{P}_{s} \subseteq\left\{x \mid \hat{\pi}_{0}<\hat{\pi} x<\hat{\pi}_{0}+1\right\}$. $\left(\hat{\pi}, \hat{\pi}_{0}\right)$ is then the required disjunction.

Conversely, suppose there exists some $\left(\hat{\pi}, \hat{\pi}_{0}\right) \in \mathbb{Z}^{n+1}$ such that $\mathcal{P}_{s} \subseteq\left\{x \mid \hat{\pi}_{0}<\hat{\pi} x<\hat{\pi}_{0}+1\right\}$. Then, $\hat{\pi}_{0}<\hat{\pi} v^{i}<\hat{\pi}_{0}+1, i=1,2, \ldots,(n+3)$. This also means that $\left|\hat{\pi}\left(v^{i}-v^{k}\right)\right|<1, i=$ $1,2, \ldots, n-1, k=1,2, \ldots, n-1$. Substituting the coordinates of $v^{i}$ and $v^{k}$, one gets: $\left|\frac{\hat{\pi}_{i}-\hat{\pi}_{k}}{2}\right|<1$. Because $\hat{\pi}_{i}, \hat{\pi}_{k} \in \mathbb{Z}$, this means that $\left|\hat{\pi}_{i}-\hat{\pi}_{k}\right| \leq 1$ for each pair $(i, k) \in\{1,2, \ldots, n-1\}^{2}$. This means that $\hat{\pi}_{i} \in\{t, t+1\}, i=1,2, \ldots, n-1$ for some $t \in \mathbb{Z}$. Let $K=\left\{i \in S \mid \pi_{i}=t+1\right\}$. Substituting the coordinates of $v^{1}$ and $\hat{\pi}$ into the inequalities $\hat{\pi}_{0}<v^{1} \hat{\pi}<\hat{\pi}_{0}+1$, one gets:

$$
\begin{gathered}
\hat{\pi}_{0}<\sum_{i=1}^{n+1} \frac{\hat{\pi}_{i}}{2 n}+\frac{\hat{\pi}_{1}}{2}<\hat{\pi}_{0}+1 \\
\Rightarrow \hat{\pi}_{0}<\frac{t}{2}+\sum_{i \in K} \frac{1}{2 n}+\frac{\hat{\pi}_{1}}{2}<\hat{\pi}_{0}+1 .
\end{gathered}
$$

Since $\frac{\hat{\pi}_{1}}{2} \in\left\{\frac{t}{2}, \frac{t+1}{2}\right\}$, the only integer value of $\hat{\pi}_{0}$ that satisfies the above condition is $\hat{\pi}_{0}=t$. Thus, $\hat{\pi} \in\{t, t+1\}^{n}, \hat{\pi}_{0}=t$. Also, $K=\phi$ would mean that $\hat{\pi}_{0}<t<\hat{\pi}_{0}+1$. This is not possible for any integers $t, \hat{\pi}_{0}$. Hence, $K$ is not empty. The condition $\hat{\pi}_{0}<\hat{\pi} v^{n}<\hat{\pi}_{0}+1$ implies:

$$
\begin{aligned}
\hat{\pi}_{0}<\sum_{i=1}^{n-1} \hat{\pi}_{i} \tilde{a}^{i}+\hat{\pi}_{n}+\hat{\pi}_{n+1}<\hat{\pi}_{0}+1 \\
\Rightarrow t<t \sum_{i=1}^{n-1} \tilde{a}^{i}+\sum_{i \in K} \tilde{a}^{i}+\hat{\pi}_{n}+\hat{\pi}_{n+1}<t+1 \\
\hat{\pi}_{n+1}=-\hat{\pi}_{n}
\end{aligned}
$$

The condition $\hat{\pi}_{0}<\hat{\pi} v^{n+1}<\hat{\pi}_{0}+1$ implies:

$$
\begin{aligned}
& t<\sum_{i=1}^{n-1} \hat{\pi}_{i} \tilde{a}^{i}+\hat{\pi}_{n}\left(\frac{1}{2}-\epsilon\right)<t+1 \\
& \Rightarrow 0<\sum_{i \in K} \tilde{a}^{i}+\hat{\pi}_{n}\left(\frac{1}{2}-\epsilon\right)<1 .
\end{aligned}
$$

The condition $\hat{\pi}_{0}<\hat{\pi} v^{n+2}<\hat{\pi}_{0}+1$ implies:

$$
\begin{aligned}
& t<t+\sum_{i \in K} \tilde{a}^{i}+\hat{\pi}_{n+1}\left(\frac{1}{2}-\epsilon\right)<t+1 \\
\Rightarrow & 0<\quad \sum_{i \in K} \tilde{a}^{i}-\hat{\pi}_{n}\left(\frac{1}{2}-\epsilon\right)<1 .
\end{aligned}
$$


Finally, the condition $\hat{\pi}_{0}<\hat{\pi} v^{n+3}<\hat{\pi}_{0}$ gives:

$$
\begin{aligned}
& t<\frac{\hat{\pi}_{n}}{2}<t+1 \\
\Rightarrow & \hat{\pi}_{n}=2 t+1 .
\end{aligned}
$$

Since the disjunction $\left(\hat{\pi}, \hat{\pi}_{0}\right)$ is the same as the disjunction $\left(-\hat{\pi},-\hat{\pi}_{0}-1\right)$, we assume without loss of generality that $\hat{\pi}_{n} \geq 0$. The condition $\hat{\pi}_{n}=2 t+1$ implies that $\hat{\pi}_{n} \geq 1, t \geq 0$. These, along with the conditions $K \neq \phi, M>3$, and equation (13) imply that $\hat{\pi}_{n}=1$. This along with equations $(13,14)$ gives,

$$
\begin{aligned}
& \sum_{i \in K} \tilde{a}^{i}<\frac{1}{2}+\epsilon \text { and } \\
& \sum_{i \in K} \tilde{a}^{i}>\frac{1}{2}-\epsilon .
\end{aligned}
$$

These conditions, along with the choice of $\epsilon$, imply respectively that $\sum_{i \in K} \tilde{a}^{i} \leq \frac{1}{2}$ and $\sum_{i \in K} \tilde{a}^{i} \geq \frac{1}{2}$. Therefore, $\sum_{i \in K} \tilde{a}^{i}=\frac{1}{2}$ and $K$ is the desired subset of $S$.

To complete the proof, we show that a description of $\mathcal{P}_{s}$ in the form $\left(P_{b}\right)$ can be obtained in polynomial time from the finite list of points $v^{1}, v^{2}, \ldots, v^{n+2}$. Note that the convex hull of $v^{1}, v^{2}, \ldots, v^{n+2}$ is a simplex in $(n+1)$ dimensions (say $Q$ ), and can be expressed in form $\left(P_{b}\right)$ in time polynomial in the size of the input as follows. If the point $v^{n+3} \in Q$, then $\mathcal{P}_{s}=Q$. Otherwise, delete from $Q$ any such inequalities that are violated by $v^{n+3}$. Call this description $\mathcal{P}^{\prime}$. Consider each of the $\frac{1}{2}(n+1)(n+2)$ hyperplanes passing through $v^{n+3}$ and any $n$ extreme points of $Q$. If all of the extreme points of $Q$ lie on one side of this hyperplane, add this to the description $\mathcal{P}^{\prime}$. Once all such hyperplanes are considered, the region $\mathcal{P}^{\prime}$ is the same as $\mathcal{P}_{s}$. This process takes time polynomial in the size of the input and yields a description of $\mathcal{P}_{s}$ in form $\left(P_{b}\right)$. The proof is now complete.

The proof provided above is not sufficient to prove a similar result for the restriction of Problem 5 in which $\pi \in\{0,1\}^{n}$ because one of the components of the vector $\pi$ in the proof above is restricted to the value of -1 . However, the following proof shows that the problem remains $\mathcal{N P}$-complete even in the presence of this restriction. The proof uses a reduction of the ONEIN-THREE-3SAT problem [Garey and Johnson, 1979; Schaefer, 1978], which is known to be $\mathcal{N} \mathcal{P}$ complete, to this problem.

Problem 6 (ONE-IN-THREE-3SAT [Garey and Johnson, 1979]). Given a set $U$ of variables and a collection $C$ of clauses over $U$ such that each clause $c \in C$ has $|c|=3$ and $c$ does not contain a negated literal. Is there a truth assignment for $U$ such that each clause in $C$ has exactly one true literal?

Problem 7 (Disjunctive infeasibility of binary programs using 0-1 hyperplanes). Given a mathematical program of the form $\left(P_{b}\right)$, does there exist $\hat{\pi} \in\{0,1\}^{n}, \hat{\pi}_{0} \in \mathbb{Z}$ such that the feasible region of each LP associated with the subproblems (3) created after branching on $\left(\hat{\pi}, \hat{\pi}_{0}\right)$ (with additional constraints $\left.x \in[0,1]^{n}\right)$ is empty?

Proposition 3.5. Problem 7 is $\mathcal{N} \mathcal{P}$-complete. 
Proof. We reduce Problem 6 to Problem 7 as follows. Associate variables $\hat{\pi}_{i}, i=1,2, \ldots, n-1$ with each variable $u_{i}$ in $U$ (where $n=|U|+1$ ). Let $\hat{\pi}_{i}=1$ if $u_{i}$ is assigned TRUE in a truth assignment and $\hat{\pi}_{i}=0$ otherwise. Clearly, an instance of Problem 6 has a required truth assignment if and only if $\hat{\pi}$ satisfies the following constraints,

$$
\begin{gathered}
\sum_{\left\{i \mid u_{i} \in c\right\}} \hat{\pi}_{i}=1, \quad \forall c \in C \\
\hat{\pi}_{i} \in\{0,1\}^{n}
\end{gathered}
$$

Let $A^{\hat{\pi}}$ be the coefficient matrix associated with the above program (with elements $a_{i j}=1$ if and only if clause $i$ contains variable $u_{j}, 0$ otherwise). Further let $\mathbf{1}$ denote a vector of all ones. If $\operatorname{rank}\left(A^{\hat{\pi}}\right)<\operatorname{rank}\left(A^{\hat{\pi}}, \mathbf{1}\right)$, then the system (15) is infeasible and there does not exist any truth assignment for Problem 6. Also, any such infeasibility can be detected in polynomial time by calculating the rank of the above matrices. Hence, it may be assumed that $\operatorname{rank}\left(A^{\hat{\pi}}\right)=\operatorname{rank}\left(A^{\hat{\pi}}, \mathbf{1}\right)$. It may also be assumed that the rows of $A^{\hat{\pi}}$ are linearly independent. Otherwise, one may drop a redundant row from (15) (or equivalently, a redundant clause from Problem 6). Using these facts, one can assume without loss of generality that $|C|=\operatorname{rank}\left(A^{\hat{\pi}}\right)=\operatorname{rank}\left(A^{\hat{\pi}}, \mathbf{1}\right) \leq|U|=n$.

Consider the convex hull $\mathcal{P}_{s}$ of $m=|C|+1$ points: $v^{i}, i=1,2, \ldots m \in \mathbb{R}^{n}$. Let the coordinate $j, v_{j}^{i}$, of each point $v^{i}$ assume a value 0 if $a_{i j}=0$ and a value $\frac{1}{2}$ if $a_{i j}=1, i=1,2, \ldots, m-1, j=$ $1,2, \ldots, n$. Let $v_{n}^{i}=0, i=1,2, \ldots, m-1$. Let $v^{m}$ be chosen such that $v_{j}^{m}=0, j=1,2, \ldots, n-$ $1, v_{n}^{m}=\frac{1}{2}$. There exists a $\hat{\pi} \in\{0,1\}^{n}$ such that $\mathcal{P}_{s} \subseteq\left\{x \in \mathbb{R}^{n} \mid \hat{\pi}_{0}<\hat{\pi} x<\hat{\pi}_{0}+1\right\}$ if and only if $v^{i} \in\left\{x \in \mathbb{R}^{n} \mid \hat{\pi}_{0}<\hat{\pi} x<\hat{\pi}_{0}+1\right\}, i=1,2, \ldots, m$. This is true if and only if $\left(\hat{\pi}, \hat{\pi}_{0}\right)$ satisfy the following conditions

$$
\begin{aligned}
& \pi_{0}<\frac{1}{2} \sum_{u_{j} \in c} \hat{\pi}_{j}<\pi_{0}+1, \quad c \in C \\
& \pi_{0}<\quad \frac{1}{2} \hat{\pi}_{n}<\hat{\pi}_{0}+1,
\end{aligned}
$$

or equivalently, if and only if $\left(\hat{\pi}, \hat{\pi}_{0}\right)$ satisfy the following conditions

$$
\begin{gathered}
\sum_{u_{j} \in c} \hat{\pi}_{j}=2 \pi_{0}+1, \quad c \in C \\
\hat{\pi}_{n}=2 \hat{\pi}_{0}+1 .
\end{gathered}
$$

Since $\hat{\pi} \in\{0,1\}^{n}$, the above conditions are satisfied if and only if $\hat{\pi}_{0}=0, \hat{\pi}_{n}=1$ and $\hat{\pi}$ satisfies the system of equations (15). Hence, an instance of Problem 6 has a required truth assignment if and only if $\mathcal{P}_{s} \subseteq\left\{x \mid \hat{\pi}_{0}<\hat{\pi} x<\hat{\pi}_{0}+1\right\}$ for some $\hat{\pi} \in\{0,1\}^{n}$. Since it was assumed that the rows of $A^{\hat{\pi}}$ are linearly independent, the points $v^{i}$ are also linearly independent. Hence, the dimension of $\mathcal{P}_{s}$ is exactly $m-1(=|C|)$. In order to obtain a description of $\mathcal{P}_{s}$ in the standard form (IP), one has to find $|C|$ facets of $\mathcal{P}_{s}$. This can be done by making $|C|$ sets, each with $|C|-1$ extreme points of $\mathcal{P}_{s}$ and finding a plane that passes through these. This can be done in time polynomial in the size of the input by solving $|C|$ systems of equations, each in $|C|-1$ variables. These $|C|$ facets can be used to describe $\mathcal{P}_{s}$ in standard form (IP). Thus, Problem 7 is $\mathcal{N} \mathcal{P}$-complete.

The complexity results for Problem 1 follow directly from those for Problem 3. In particular, Problem 1 is $\mathcal{N} \mathcal{P}$-hard and remains so even when the restrictions described in Proposition 3.3 are applied and even for the case of binary programs. 


\section{Related Problems}

We now show that one can directly deduce from Proposition 3.2, the complexity of the following two problems associated with verifying whether a given inequality is a split-inequality:

Problem 8. Given a mathematical program of the form $(I P)$, is a given inequality $(\alpha, \beta)$ an elementary split inequality?

Problem 9. Given a mathematical program of the form (IP) and $z_{l} \in \mathbb{R}$, does there exist a single elementary split inequality for (IP) such that the LP relaxation bound achieved after adding it is at least $z_{l}$ ?

The above two problems may appear similar to the problem of separation from the elementary split closure of (IP), a problem that has been shown to be $\mathcal{N} \mathcal{P}$-complete by Caprara and Letchford [2003]. So we first start by distinguishing these problems. It is obvious that all elementary split inequalities have split-rank at most one. Also, any inequality that is a convex combination of two elementary split inequalities has split-rank at most one. However, since two different disjunctions may have been used to generate the two elementary split inequalities thus combined, the convex combination of these inequalities may not necessarily be an elementary split inequality, even though its rank is one. As an example, consider the following system

$$
\begin{aligned}
x_{1} & \leq 0.8 \\
x_{2} & \leq 0.8 \\
x & \in \mathbb{Z}^{2} .
\end{aligned}
$$

The inequality $x_{1}+x_{2} \leq 0$ has split-rank one with respect to (16) because it can be obtained as a convex combination of the elementary split inequalities $x_{1} \leq 0$ and $x_{2} \leq 0$. The inequality $x_{1}+x_{2} \leq 0$ separates the points $\left(\frac{1}{2}, 0\right),\left(0, \frac{1}{2}\right),\left(\frac{1}{2}, \frac{1}{2}\right)$, which are in the associated polyhedron $\mathcal{P}$, from the feasible region of (16). If this were an elementary split inequality for $\mathcal{P}$ generated using a disjunction $\left(\pi, \pi_{0}\right)$, neither of the three points $\left(\frac{1}{2}, 0\right),\left(0, \frac{1}{2}\right),\left(\frac{1}{2}, \frac{1}{2}\right)$ should lie in the disjunctive subsets obtained after applying the disjunction. So, $\left(\pi, \pi_{0}\right)$ should, at least, satisfy the following three constraints:

$$
\begin{gathered}
\pi_{0}<\quad \frac{1}{2} \pi_{1}<\pi_{0}+1 \\
\pi_{0}<\quad \frac{1}{2} \pi_{2}<\pi_{0}+1 \\
\pi_{0}<\quad \frac{1}{2}\left(\pi_{1}+\pi_{2}\right)<\pi_{0}+1 \\
\pi_{0}, \pi_{1}, \pi_{2} \in \mathbb{Z} .
\end{gathered}
$$

Since the system (17) is infeasible, there is no such disjunction and hence $x_{1}+x_{2} \leq 0$ is not an elementary split inequality even though it is a convex combination of two such inequalities.

The result of Caprara and Letchford [2003] shows that the problem of determining whether a given point $x$ may be separated from the elementary split closure of (IP) is $\mathcal{N} \mathcal{P}$-complete. Using the equivalence of separation and optimization, it follows that the problem of deciding whether a given inequality has split rank one or not is $\mathcal{N} \mathcal{P}$-complete. This observation leads directly to the result that the following problem is $\mathcal{N} \mathcal{P}$-complete. 
Problem 10. Given a mathematical program of the form (IP) and $z_{l} \in \mathbb{R}$, does there exist a set $S$ of split inequalities of rank one associated with $\mathcal{P}$ such that the LP relaxation bound achieved after adding all of the inequalities in $S$ is at least $z_{l}$ ?

However, the above result does not imply anything about the complexity of showing that a given inequality is an elementary split inequality. We have already seen that even if an inequality has split rank one, it may not be an elementary split inequality. We now show that the complexity of this problem follows directly from Proposition 3.2.

Proposition 4.1. Problem 8 is $\mathcal{N} \mathcal{P}$-complete.

Proof. Consider the special case when $\alpha=0, \beta=1$. Then $\alpha x \geq \beta$ (or $0 \geq 1$ ) is a split inequality for (IP) if and only if there exists a disjunction $\left(\hat{\pi}, \hat{\pi_{0}}\right)$ such that the associated LPs $(3)$ are infeasible. By Proposition 3.2, the problem of finding such $\left(\hat{\pi}, \hat{\pi}_{0}\right)$ is $\mathcal{N} \mathcal{P}$-complete.

In contrast, consider the case of Chvátal-Gomory (C-G) inequalities. Given a pure integer program (IP), a C-G inequality for (IP) is an inequality of the form

$$
\alpha x \geq\lceil\beta\rceil,
$$

where $\alpha \in \mathbb{Z}^{n}$ and $\alpha x \geq \beta$ is a valid inequality for the feasible region $\mathcal{P}$ of the LP relaxation. Eisenbrand [1999] showed that the problem of separating a given point $x$ from the elementary C-G closure of (IP) is $\mathcal{N} \mathcal{P}$-complete. Hence, the problem of deciding whether a given inequality has $\mathrm{C}-\mathrm{G}$ rank one or not is also, like for the case of split inequalities, $\mathcal{N} \mathcal{P}$-complete. However, unlike split inequalities, we can decide whether a given inequality (say $\alpha x \geq \delta$, where $\alpha \in \mathbb{Z}^{n}, \delta \in \mathbb{Z}$ ) is an elementary C-G inequality or not in polynomial time by solving the LP

$$
\begin{array}{r}
\min \alpha x \\
A x \geq b .
\end{array}
$$

Then, $\alpha x \geq \delta$ is an elementary C-G inequality for (IP) if and only if the optimal solution to (19) is strictly greater than $\delta-1$.

The above results seem somewhat surprising. On the one hand, the problem of deciding whether the rank of a given inequality is one lies in the complexity class $\mathcal{N} \mathcal{P}$-complete for the case of both $\mathrm{C}-\mathrm{G}$ inequalities and split inequalities. On the other hand, the problem of deciding whether a given inequality is an elementary $\mathrm{C}-\mathrm{G}$ inequality lies in complexity class $\mathcal{P}$, while the same problem for a split inequality lies in complexity class $\mathcal{N} \mathcal{P}$-complete.

Proposition 4.2. Problem 9 is $\mathcal{N} \mathcal{P}$-complete.

Proof. The proof follows directly from Proposition 3.2 and Proposition 2.1.

It is also well known that one can always find an elementary split inequality (for instance, a Gomory mixed integer inequality) to separate a given basic feasible solution of the LP relaxation, that is not feasible for the original problem, from the elementary split closure in time polynomial in size of the input (see, for instance, Cornuéjols [2008]). However, the above problem of maximizing the lower bound by adding a valid split inequality remains $\mathcal{N} \mathcal{P}$-hard even if an optimal basic feasible point of $\mathcal{P}$ is provided as an input because the desired inequality must separate the set of all points that have lower objective values regardless of whether they are basic feasible solutions. 


\section{Conclusions}

In this paper, we showed that the problem of selecting a general disjunction whose imposition causes the LP relaxation become infeasible (either for branching or for generating an elementary split inequalities) is $\mathcal{N} \mathcal{P}$-complete. This leads to two important results - that the problem of selecting a general disjunction that maximizes the bound improvement (again, both by branching or generating an elementary split inequality) of a given IP is $\mathcal{N} \mathcal{P}$-hard, and that the problem of deciding whether a given inequality is an elementary split inequality is $\mathcal{N} \mathcal{P}$-complete. We also showed that the first problem remains $\mathcal{N} \mathcal{P}$-hard even when several natural restrictions are imposed on the disjunctions or when all integer-constrained variables in the IP are binary. Only a few studies have exploited the relationship between disjunctions used for generating split inequalities and for branching. We believe that this topic deserves more attention. There is also a need to develop fast heuristics to solve the problem of selecting good disjunctions so as to enhance the speed of branch-and-bound and cutting-plane algorithms.

Acknowledgements: The authors would like to acknowledge the anonymous referees whose suggestions led to a considerable improvement in the presentation of the paper.

\section{References}

K. Aardal and F. Eisenbrand. Integer programming, lattices and results in fixed dimenstion. Technical report, Probability, Networks and Algorithms, 2004.

T. Achterberg, T. Koch, and A. Martin. Branching rules revisited. Operation Research Letters, 33: 42-54, 2005.

E. Balas and A. Saxena. Optimizing over the split closure. Mathematical Programming, Series A, 113(2):219-240, 2008.

W. Banaszczyk, A. E. Litvak, A. Pajor, and S. J. Szarek. The flatness theorem for nonsymmetric convex bodies via the local theory of banach spaces. Mathematics of Operations Research, 24(3): 728-750, 1999.

A. Caprara and A. N. Letchford. On the separation of split cuts and related inequalities. Mathematical Programming, Series B, 94:279-294, 2003.

W. Cook, R. Kannan, and A. Schrijver. Chvátal closures for mixed integer programming problems. Mathematical Programming, 47:155-174, 1990.

G. Cornuéjols. Valid inequalities for mixed integer linear programs. Mathematical Programming, 112(1):3-44, March 2008.

G. Cornuéjols, L. Liberti, and G. Nannicini. Improved strategies for branching on general disjunctions. Working Paper, 2008.

I. Derpich and J. R. Vera. Improving the efficiency of branch and bound algorithm for integer programming based on "flatness" information. European Journal of Operational Research, 174: 92-101, 2006. 
F. Eisenbrand. Note on the membership problem for the elementary closure of a polyhedron. Combinatorica, 19(2):297-300, 1999.

M. Fischetti and A. Lodi. Local branching. Mathematical Programming, 98:23-47, 2003. Series B.

M. R. Garey and D. S. Johnson. Computers and Intractability: A guide to the theory of NPcompleteness. W.H. Freeman and Co., 1979.

A. Gilpin and T. Sandholm. Information-theoretic approaches to branching in search. In IJCAI, pages 2286-2292, 2007.

R. E. Gomory. Outline of an algorithm for integer solutions to linear programs. Bulletin of American Mathematical Society, 64:275-278, 1958.

J. H.W. Lenstra. Integer programming with a fixed number of variables. Mathematics of Operations Research, 8:538-548, 1983.

M. Karamanov and G. Cornuéjols. Branching on general disjunctions. Working Paper, 2007.

B. Krishnamoorthy. Is Thinner Better. Working Paper, 2008.

B. Krishnamoorthy and G. Pataki. Column basis reduction and decomposable knapsack problems, 2006. Submitted, available at http://www.optimizationonline.org/DB_HTML/2007/06/1701.html.

P. Liberatore. On the complexity of choosing the branching literal in DPLL. Artificial intelligence, 116(1-2):315-326, January 2000.

J. Linderoth and M. Savelsbergh. A computational study of search strategies for mixed integer programming. INFORMS Journal on Computing, 11:173-187, 1999.

A. Mahajan and T. K. Ralphs. Experiments with branching using general disjunctions. In J. W. Chinneck, B. Kristjansson, and M. J. Saltzman, editors, Operations Research and CyberInfrastructure, volume 47, 2009.

G. L. Nemhauser and L. A. Wolsey. Integer and Combinatorial Optimization. John Wiley \& Sons, Inc., 1988.

J. H. Owen and S. Mehrotra. Experimental results on using general disjunctions in branch-andbound for general-integer linear programs. Computational optimization and applications, 20(2), November 2001.

J. Patel and J. W. Chinneck. Active constraint variable ordering for faster feasibility of mixed integer linear programs. Mathematical Programming, 110(3):445-474, September 2007.

T. J. Schaefer. The complexity of statisfiability problems. In Proceedings of the tenth annual ACM symposium on Theory of computing, pages 216-226, 1978.

A. Sebő. An introduction to empty lattice simplices. In Proceedings of the 7th International IPCO Conference on Integer Programming and Combinatorial Optimization, LNCS, pages 400-414, 1999. 